\title{
Case of a Rare Variant of Deep Cerebral Venous Thrombosis after Treatment with Norgestrel-Ethinyl Estradiol
}

\author{
Swapna Bhaskar ${ }^{1}$, Alevoor Rajaram Bhat ${ }^{2}$, Badakare Chidamber Rao ${ }^{3}$ \\ ${ }^{1}$ Department of Family Medicine, St Philomena's Hospital, Bengaluru, Karnataka, INDIA. \\ ${ }^{2}$ Department of Neurology, St Philomena's Hospital, Bengaluru, Karnataka, INDIA. \\ ${ }^{3}$ Family Physician, $7^{\text {th }}$ cross, Indiranagar, Bengaluru, Karnataka, INDIA.
}

\begin{abstract}
Cerebral Venous Thrombosis (CVT) is a rare but potentially life-threatening disease characterized by thrombosis of the cerebral venous system, frequently seen in young population. Use of Oral Contraceptive Pills (OCP) is one of the major risk factors for CVT in women along with pregnancy, collagen vascular diseases and other hypercoagulable states. The symptoms and signs of CVT can be quite vague like headache and requires quick assessment and investigations like magnetic resonance imaging with angiogram for diagnosis. Although complete recovery is possible with early treatment, mortality can be seen in as much as $15 \%$ of cases if treatment is delayed. This article discusses a very rare variety of CVT and reviews the epidemiology, risk factors, clinical presentation, diagnosis and management of CVT.
\end{abstract}

Key words: Oral Contraceptive Pills, Cerebral venous thrombosis, PCOD.

\section{INTRODUCTION}

CVT can occur in females using hormonal pills, many a time presenting with masquerading symptoms. Deep cerebral venous thrombosis (DCVT) is a rare form seen in only $10 \%$ of cases. We report a case of DCVT in a teenaged girl following short term use of norgestrel- ethinyl estradiol prescribed by her Gynecologist for control of PCOD related menorrhagia.

\section{CASE REPORT}

This 16-year-old girl presented to the ER with generalized weakness and headache for 7 days which exacerbated since few hours causing abnormal sensorium. She was undergoing treatment from a gynecologist for irregular menstrual cycles and menorrhagia for which she was taking norgestrel-ethinyl estradiol tablets (diagnosed as PCOD).

Her headache was very severe associated with vomiting, photophobia and prostration.
On examination patient was afebrile with normal vitals, but restless and disoriented with severe pallor. There was no focal neurological deficit or signs of meningeal irritation. During hospitalization, she developed generalized tonic clonic seizures which was treated with IV levirecetam. Her blood investigations showed HB of $5.5 \mathrm{gm}$ while other cell counts, RFT, LFT and TSH were normal. CT brain was done immediately which showed straight sinus and deep cerebral vein thrombosis. (Figure 1). She was started on Low Molecular Weight Heparin (LMWH) along with other symptomatic treatment. MR venogram showed bilateral symmetrical recent thalamic infarcts with involvement of left caudate nucleus, putamen, basi temporal lobe and right tegmentum due to thrombosis of internal cerebral veins, vein of Galen, straight sinus and left veins of label (Figure 2).
DOI: 10.5530/ijopp.12.1.9

Address for correspondence: Dr Swapna Bhaskar, HOD- family medicine, St Philomena's Hospital, No 4, Campbell Road, Neelasandra, Bengaluru47, Karnataka, INDIA. Phone no: +919886079418 Email Id: swapnabhaskar305@ gmail.com

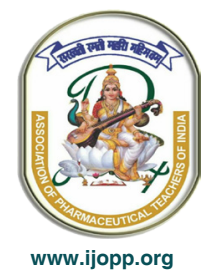




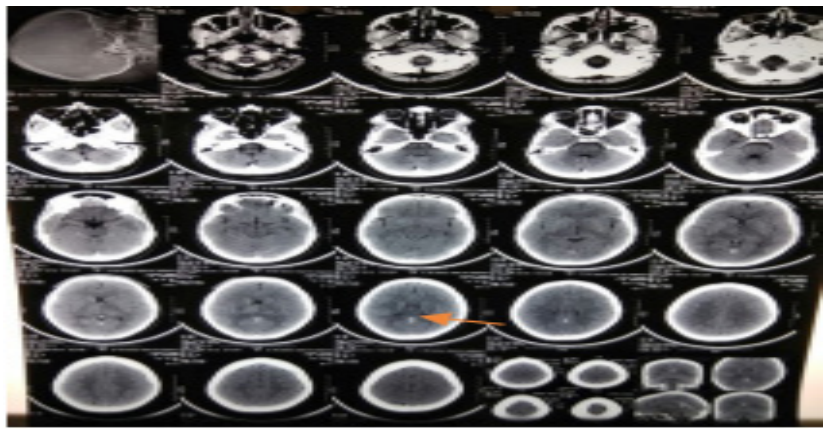

Figure 1 : Explained with Arrows to Abnormal Area.

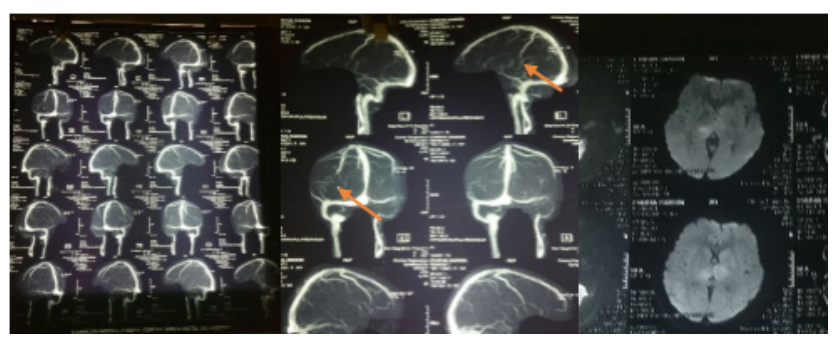

Figure 2 : Explained with Arrows.

Prothrombin Time, INR and ANA profile were normal and she was started on acicoumarin and dose adjusted to maintain INR 2-3. Her headache resolved in 3 days, sensorium returned to full normalcy. Patient was also transfused 3 units of packed cells during the stay. She was discharged on day 7 with anticoagulants, iron supplements and antiepileptic medication with advice for regular PT/ INR monitoring. There was no further menorrhagia, but she was asked to follow lifestyle changes and advised tranexamic acid if menorrhagia recurs. Further workup for other causes of CVT like coagulation profile, protein $\mathrm{C}$ and $\mathrm{S}$, antithrombin 3 etc. were deferred to a later date since she had to be started on heparin in an emergent situation. There was consensus among the treating team regarding the necessity of treatment first and the most probable cause of the DCVT being her hormonal pill usage. She was on regular follow up maintaining desired INR for 6 months after which her repeat MR venogram showed normal sinuses. Coagulation profile was done after stopping the anticoagulants which showed normal PT, APTT, Protein C, Protein S, antithrombin 3 and negative APLA. Hence the diagnosis of drug induced CVT was confirmed and patient was advised to avoid OCPs throughout life and treat her PCOD symptomatically and with lifestyle measures.

\section{DISCUSSION}

The incidence of CVT is around 3 - 4 cases per one million population, common in adults between
20-40 years showing female preponderance. ${ }^{1}$ Although there are no population-based studies in India, some hospital-based studies have shown CVT to be the cause in $10 \%$ of stroke cases in young. ${ }^{2}$

Thrombosis can occur in superficial veins, deep venous system or cortical veins of brain. Superior sagittal and transverse sinuses are the most commonly affected, followed by sigmoid sinus, straight sinus, cortical veins, deep cerebral and internal cerebral veins. The term Deep cerebral vein thrombosis (DCVT) is used for thrombosis of internal cerebral vein, vein of Galen, basal vein of Rosenthal and its tributaries and is relatively rare with incidence in only $10 \%$ cases. ${ }^{3,4}$ Our patient had DCVT involving straight sinus, vein of Galen and internal cerebral vein as shown in the MR venogram.

Various risk factors for CVT includes pregnancy, OCPs, hyperhomocystenemia, CNS infections, autoimmune diseases, trauma, malignancy, infections, thrombophilia, dehydration and congenital or acquired abnormalities of the hemostatic system. ${ }^{5}$

The common clinical features are headache, papilledema, altered sensorium, seizures, sensorimotor deficits, decreased consciousness and hemorrhage. Severe presentations include hemiparesis or aphasia due to bilateral or unilateral edema and hemorrhagic infarction of the basal ganglia and thalami. ${ }^{6}$

CVT is three times more prevalent in women taking oral contraceptive pills (OCPs). A recent metanalysis found a 7.59 time increase in incidence among women on $\mathrm{OCPs}^{7}$ but did not find the answer on the duration and forms of pills. Many studies suggest increased chances in patients taking third generation OCPs. The pills used by our patient contained Norgestrel and ethinyl estradiol - a second generation OCP.

The pathology for this increased incidence is multifactorial. The changes of the hemostatic system include stimulated procoagulation, inhibited anticoagulation and elevated fibrinolytic activity. The latter is caused by elevated plasminogen level and a higher tissue plasminogen activator (tPA) activity. The plasminogen activator inhibitor I (PAI - I) antigen and activity also decrease. ${ }^{8}$ The WHO recommends avoiding using OCPs in women who have thrombogenic mutations, smokers, those aged 35 years or more and 21 days post-partum to prevent thromboembolic complications. ${ }^{9}$

Women who have suffered CVT with use of OCP should seek alternative non-estrogen-based methods for contraception like the progestin-only pill, levonorgestrel 
intrauterine device or copper intrauterine device. Those with a history of CVT during pregnancy or during the postpartum period have an increased risk of recurrence during subsequent pregnancies and hence prophylactic anticoagulation with low-molecular weight heparin during future pregnancies and the postpartum period is recommended.

The American Stroke Association (ASA) recommends imaging of the cerebral venous system in patients with suspected CVT. ${ }^{10}$ Noncontrast head CT may demonstrate venous infarcts or hemorrhages or alternative diagnoses, but it has poor sensitivity. CT signs are hyper density in the area of a sinus or cortical vein (cord sign) and filling defects, especially in the superior sagittal sinus (empty sign) in contrast-enhanced studies. Our patient's CT showed hyder density of straight sinus and deep cerebral veins with filling defects in contrast induced study.

Magnetic resonance imaging with MR venography is more conclusive. It shows abnormal signal intensity in the sinus with absence of flow but is contraindicated in patients with renal impairment because of the requirement of gadolinium contrast and the associated risk of nephrogenic systemic fibrosis. MR venogram in our patient revealed absence of flow signals in straight sinus, internal cerebral vein, vein of Galen and left vein of Labbe, suggestive of thrombosis.

Treatment consists of heparin, anticoagulation and in some cases thrombolytic therapy. The overall prognosis of CVT is favorable but, further research is needed to outline optimal treatment options including the duration of anticoagulation therapy and the role of thrombolytic therapy. The thirty-day mortality rate is $3-15 \%$ and more than $75 \%$ of patients experience complete recovery within six to twelve months. The most common cause of death during the acute phase of CVT is Tran's tentorial herniation, most frequently from large venous hemorrhage.

Our patient was treated with enoxaparin for the acute phase and followed up with acicoumarin to maintain her INR in the desired range. Her menorrhagia has not recurred since admission, but irregular menstrual cycles were persisting. She was advised lifestyle modification including exercises and tranexamic acid for immediate use in case of menorrhagia.

\section{CONCLUSION}

CVT is a rare but potentially life threatening adverse effect of treatment with OCPs. Awareness, immediate investigations and diagnosis by the primary care physicians and/or gynecologists can be lifesaving for suspected cases even though patient may present with vague symptoms. Diagnosis in this patient was done with $\mathrm{CT}$ and MR venogram and early suspicion and immediate use of LMWH and anticoagulation helped in her complete recovery.

\section{CONFLICT OF INTEREST}

The authors declare no conflict of interest.

\section{REFERENCES}

1. Filippidis A, Kapsalaki E, Patramani G, Fountas KN. Cerebral venous sinus thrombosis: Review of the demographics, pathophysiology, current diagnosis and treatment. Neurosurgical Focus. 2009;27(5):E3. doi: 10.3171/2009.8. FOCUS09167.

2. Narayan D, Kaul S, et al. Risk factors, clinical profile and long-term outcome of 428 patients of cerebral sinus venous thrombosis: Insights from Nizam's Institute Venous Stroke Registry, Hyderabad (India). Neurol India. 2012;60(2):154-9.

3. Ferro JM, Canhão P, Stam J, Bousser MG, Barinagarrementeria F. Prognosis of cerebral vein and Dural sinus thrombosis: Results of the International Study on Cerebral Vein and Dural Sinus Thrombosis (ISCVT). Stroke: A Journal of Cerebral Circulation. 2004;35(3):664-70.

4. Prabhat K, Gargi S, Subodh KM, Shreya G, Harish G. Deep Cerebral Vein Thrombosis: A Clinical Masquerader. Journal of Clinical and Diagnostic Research. 2017;11(4):OD1-16. DOI: 10.7860/JCDR/2017/25910.9712

5. McBane RD, Tafur A, Wysokinski WE. Acquired and congenital risk factors associated with cerebral venous sinus thrombosis. Thrombosis Research. 2010;126(2):81-7.

6. Ehtisham A, Stern BJ. Cerebral venous thrombosis: A review. The Neurologist. 2006;12(1):32-8.

7. Payam K, Kambiz H. A case-series study of cerebral venous thrombosis in women using short course oral contraceptive. Iran J Neurol. 2016;15(2):92-5.

8. Margaret AR, Eric MD. Two cases of venous sinus thrombosis in women taking the oral contraceptive pill, Yaz (dropirenone/ ethinyl estradiol) and review of the literature. Health. 2013;5(07):92-9.

9. World Health Organization. Medical eligibility criteria for contraceptive use. 2008. Update. http://whqlibdoc.who.int/hq/2008/WHO_RHR_08.19_eng.pdf 10. Saposnik G, Barinagarrementeria F, Brown RDJ, Bushnell CD, Cucchiara $\mathrm{B}$, Cushman M, et al. American Heart Association Stroke Council and the Council on Epidemiology and Prevention. Diagnosis and management of cerebral venous thrombosis: a statement for healthcare professionals from the American Heart Association/American Stroke Association. Stroke. 2011;42(4):1158-92. 